\title{
Competitive exclusion and coexistence phenomena of a two-strain SIS model on complex networks from global perspectives
}

\section{Xiaoyan Wang ${ }^{1} \cdot$ Junyuan Yang $^{2,3}$ (D) Xiaofeng Luo ${ }^{4}$}

Received: 8 November 2021 / Revised: 17 January 2022 / Accepted: 27 January 2022 /

Published online: 16 February 2022

(c) The Author(s) under exclusive licence to Korean Society for Informatics and Computational Applied Mathematics 2022

\begin{abstract}
Genetic heterogeneity plays an important role in exploring the interplays of microorganisms. Competitive exclusion principle is the main principle that governs causative agentries of diseases competition. Identifying coexistence mechanisms is a core issue for studying the interactions of multi-strains. In this paper, we are concerned with the dynamics of a two-strain SIS epidemic model with general incidence rate on complex networks. We derive the basic reproduction numbers and the invasion reproduction numbers associated with each strain, which determine the competitive, exclusion and coexistence of the two strains. We strictly prove that the competitive exclusion principle holds in a global sense and the endemic equilibrium coexists uniquely and globally. Numerical examples visibly illustrate the evolution of the two strains.
\end{abstract}

Keywords Competitive exclusion principle $\cdot$ Global perspectives $\cdot$ Complex networks

These authors contributed equally to this work.

$凶 \quad$ Junyuan Yang

yjyang66@sxu.edu.cn

1 School of Information, Shanxi University of Finance and Economics, Wucheng Road, Taiyuan 030006, Shanxi, China

2 Complex Systems Research Center, Shanxi University, Wucheng Road, Taiyuan 030006, Shanxi, China

3 Shanxi Key Laboratory of Mathematical Techniques and Big Data Analysis on Disease Control and Prevention, Shanxi University, Wucheng Road, Taiyuan 030006, Shanxi, China

4 School of Science, North University of China, Xueyuan Road, Taiyuan 030051, Shanxi, China 


\section{Introduction}

Infectious diseases are essentially caused by bacteria, virus and parasites. Infectious agents are represented by multiple variants and often denoted by strains. Many diseases are indeed caused by multiple strains of a pathogen, such as dengue, influenza, malaria and COVID-19, etc, and the dominant strain alters during an outbreak. Multi-strain convolves and complicates the process of the epidemic and increases the difficulties of control. Mathematical modeling provides a theoretical insight for investigating the dynamics of the coevolution of multiple strains. Overall, the aim of muti-strain models is to investigate the competing and co-existence outcomes in varying significant epidemiological parameters, such as transmission probability and duration of infectious period, etc.

Competitive exclusion is an important principle in theoretical biology, which suggests that no two species can forever occupy the same ecological niche [1,2]. Many scholars have brought such theory to investigate the evolutionary interactions of host and pathogen populations [3-7]. In view of existing results, the competitive exclusion principle and coexistence are two main projects for studying the interplays of multiple strains, which are in favor of taking effective control measures for policymakers. From epidemiological view of points, the competitive exclusion principle shows that one of strains dominates and the other strains burn out. Mathematically speaking, one variable associated with one strain attends to a certain constant and the other variables corresponding to other strains approach zero as the time goes to infinity. Surely, some multi-strain models exhibit competitive exclusion phenomena. For instance, Bremermann and Thieme [8] considered a multi-strain SIR model, which displays such phenomena i.e, only one strain with the largest reproduction number survives. Martcheva and her collaborators [9, 10] investigated a multi-strain vector-borne disease model and a multi-strain flu model and both of them serve as that competitive exclusion results. On the contrary, many scholars are conducted to digging out some mechanisms, such as mutation [11], superinfection [12], cross-immunity [13], and nonlinear incidence rate [14], etc., to make multiple strains coexist.

However, all the models mentioned above are assumed that populations are homogeneously mixed and they ignore the contact heterogeneities. Often, these models overestimate partnerships of contacts and thus they may correctly characterize multistrain epidemics prevalence in large-scale social contact networks. Hence, much effort has been attracted to concern with the multi-strain epidemic models on complex networks, which describes contact heterogeneities among individuals. Karrer and Newman considered a model of two competing diseases spreading on a static network and they showed that competitive exclusion and coexistence may happen [16]. Wu et al. proposed a mean-field SIS model with two strain on complex networks and strictly analyzed the dynamics of each feasible equilibrium [17]. Yang et al. created a multi-strain SIS epidemic model with non-markovian process on complex networks and found the competitive and exclusion phenomena [18]. In [19, 20], Wu and his collaborators found that mutation and superinfection are two main factors resulting in coexistence of two strains on scale free networks. Yang and Li identified that a saturation incidence rate is one of mechanisms leading to coexistence of two strains [21]. Huang et al, extended that incidence rate as a general one and found the similar 
outcomes. In [23], Chen et al. constructed a pairwise model to describe the competitive exclusion regime by the heterogeneous mean-field approach. Lv and Jin investigated competitive exclusion phenomena in forms of final size associated with each strain by an edge-compartmental multi-strain model on complex networks [24]. However, most of competitive and exclusion results are viewed in a local sense not in a global perspective [22]. In fact, the competitive exclusion principle should be globally established or it has to hold for all values of the initial conditions from mathematical points of view [25].

The main focuses of this paper includes two aspects: one is that we rigorously solve the competitive exclusion outcomes in a global sense by constructing smart Lyapunov functions. The other one is to capture the existence and global stability of coexistence equilibrium by using invasive theory and constructing an analogous Lyapunov function as the first focus.

The organization of this paper is taken as follows: Sect. 2 presents a degree-based two-strain SIS epidemic model with nonlinear incidence and it is further translated into a mixed degree-edge model by an aggregating approach. In Sect. 3, the main theoretical results, such as the competitive exclusion and coexistence phenomena have been established. In Sect. 4, some applications and numerical simulations have been vividly illustrated.

\section{The model formula}

As mentioned in [21, 22], the total nodes in a complex network are categorized by three different statuses. Let $s_{k}(t), \rho_{k}(t)$ and $\vartheta_{k}(t)$ represent the densities of susceptibles, densities of nodes infected by strain one and densities of nodes infected by strain two, respectively. Susceptibles can be both infected by strain one and strain two. Infected individuals recover by themselves or treated and then move back to the susceptible class. The two-strain model with a competing mechanism is taken in the following form:

$$
\left\{\begin{array}{rl}
\frac{d s_{k}(t)}{d t}= & -\beta_{1} k s_{k} F_{1}\left(\Theta_{1}\right)-\beta_{2} k s_{k} F_{2}\left(\Theta_{2}\right)+\gamma_{1} \rho_{k} \\
& +\gamma_{2} \vartheta_{k}, \\
\frac{d \rho_{k}(t)}{d t}= & -\gamma_{1} \rho_{k}(t)+\beta_{1} k s_{k} F_{1}\left(\Theta_{1}\right), \\
\frac{d \vartheta_{k}(t)}{d t}= & -\gamma_{2} \vartheta_{k}(t)+\beta_{2} k s_{k} F_{2}\left(\Theta_{2}\right),
\end{array} \quad k=1,2, \cdots, n\right.
$$

where $\beta_{i}$ and $\gamma_{i}$ for $i=1,2$ stand for the transmission rate and recover rate, respectively. The formula

$$
\Theta_{j}(t)=\frac{1}{\langle k\rangle} \sum_{k=1}^{n} k p(k) l_{k}(t), l=\rho, \vartheta
$$

denotes the probability of an susceptible node connecting other infected nodes. The nonlinear function $F_{j}(j=1,2)$ have the following properties: for $\Theta_{j} \geq 0$, 
(1) $F_{j}\left(\Theta_{j}\right) \geq 0$, and $F_{j}(0)=0$;

(2) $F_{j}^{\prime}\left(\Theta_{j}\right)>0$ and $F_{j}^{\prime \prime}\left(\Theta_{j}\right) \leq 0$;

(3) $\frac{F_{j}\left(\Theta_{j}\right)}{\Theta_{j}}$ is a decreasing function for any $\Theta_{j} \in[0,1)$.

It is not hard to see that if $F_{j}\left(\Theta_{j}\right)=\frac{\Theta_{j}}{1+\alpha_{j} \Theta_{j}}, j=1,2$, then model (1) is same as the model proposed by Yang and Li [21]; if $k F_{j}\left(\Theta_{j}\right)=F_{k j}\left(\Theta_{j}\right), j=1,2, k=$ $1,2, \cdots, n$, then it becomes the model proposed by Huang et al. [22].

For the convenience, let $\gamma_{1}=\gamma_{2}=\gamma$. Then we adopt the method proposed by Wang and Yang [26] to aggregate (2) into

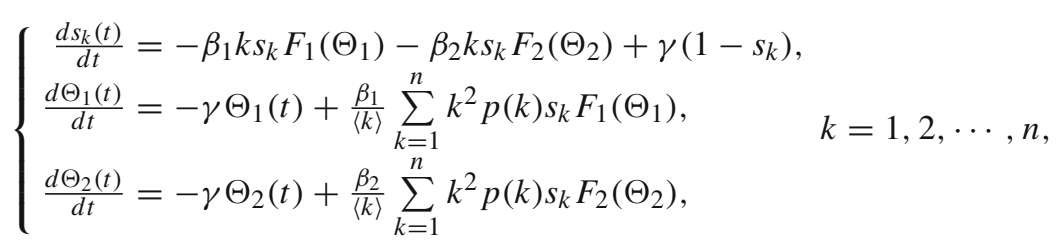

In what follow, we are concerned with the dynamics of Eq.(2) instead of Eq.(1). The following theorem shows the property of its solution.

Theorem 1 For any initial data $\phi=\left(s_{k 0}, \Theta_{10}, \Theta_{20}\right) \in\left(\mathbb{R}_{+}\right)^{n+2}$, the solutions of system (2) preserve nonnegative i.e, for all $t \in \mathbb{R}_{+}$,

$$
s_{k}(t)>0, \Theta_{1}(t) \geq 0, \Theta_{2}(t) \geq 0
$$

Furthermore, for any $t \in \mathbb{R}_{+}$,

$$
s_{k}(t) \leq \max _{k=1, \cdots, n}\left\{s_{k 0}, 1\right\}, \Theta_{j}(t) \leq \max \left\{\Theta_{j 0}, 1\right\}, j=1,2 .
$$

Proof The positivity of $s_{k}$ is a straightforward consequence of the positive tangent curve theory. In fact, assume that $s_{k}(t)=0$, then the first equation of (2) becomes

$$
s_{k}^{\prime}(t)=\gamma>0
$$

which implies that all the direct vector fields move into the first octant and so that for all $t \in \mathbb{R}_{+}, s_{k}(t)>0$. From Assumption (2) for $F$ it follows that $F_{j}\left(\Theta_{j}\right) \geq$ $F^{\prime}(1) \Theta_{j}(j=1,2)$. The positivity of $\Theta_{j}(j=1,2)$ is an immediate result of the second and the third equations of Sys.(2).

The nonnegativity of $s_{k}, \Theta_{j}(j=1,2)$, together with the definition of $F_{j}$, indicates that

$$
s_{k}^{\prime}(t) \leq \gamma-\gamma s_{k}(t)
$$

hence

$$
s_{k}(t) \leq\left(s_{k 0}-1\right) e^{-\gamma t}+1 .
$$


Define

$$
\Theta_{s}(t)=\frac{1}{\langle k\rangle} \sum_{k=1}^{n} k p(k) s_{k}(t) .
$$

Then

$$
\left(\Theta_{S}(t)+\Theta_{1}(t)+\Theta_{2}(t)\right)^{\prime}=\gamma-\gamma\left(\Theta_{S}(t)+\Theta_{1}(t)+\Theta_{2}(t)\right),
$$

which suggests that

$$
\left(\Theta_{S}(t)+\Theta_{1}(t)+\Theta_{2}(t)\right) \leq\left(\Theta_{0}-1\right) e^{-\gamma t}+1
$$

where $\Theta_{0}=\Theta_{s 0}+\Theta_{10}+\Theta_{20}$ and $\Theta_{s 0}=\frac{1}{\langle k\rangle} \sum_{k=1}^{n} k p(k) s_{k 0}$. Inequalities (3) and (4) admit a fact that for all $t \in \mathbb{R}_{+}$,

$$
s_{k}(t) \leq \max _{k=1, \cdots, n}\left\{s_{k 0}, 1\right\}
$$

and

$$
\Theta_{j}(t) \leq \max \left\{\Theta_{j 0}, 1\right\}
$$

This completes the proof.

By Theorem 1, we can define the following sets

$$
\begin{aligned}
& \Omega=\left\{\phi_{0} \in\left(\mathbb{R}_{+}\right)^{n+2}: 0<s_{k} \leq 1,0 \leq \Theta_{j} \leq 1, k=1,2, \cdots, n, j=1,2\right\} \\
& \Omega_{1}=\left\{\phi_{0} \in\left(\mathbb{R}_{+}\right)^{n+2}: 0<s_{k} \leq 1,0 \leq \Theta_{1} \leq 1, \Theta_{2}=0, k=1,2, \cdots, n\right\}, \\
& \Omega_{2}=\left\{\phi_{0} \in\left(\mathbb{R}_{+}\right)^{n+2}: 0<s_{k} \leq 1, \Theta_{1}=0,0 \leq \Theta_{2} \leq 1, k=1,2, \cdots, n\right\},
\end{aligned}
$$

which are positively invariant. In the following, we will investigate the long-time behaviors of system (2) taken initial values from $\Omega$.

\section{The main results}

For any feasible equilibrium $E^{*}=\left(s_{k}^{*}, \Theta_{1}^{*}, \Theta_{2}^{*}\right)$, its components satisfy the following equations

$$
\left\{\begin{array}{l}
0=-\beta_{1} k s_{k} F_{1}\left(\Theta_{1}^{*}\right)-\beta_{2} k s_{k} F_{2}\left(\Theta_{2}^{*}\right)+\gamma\left(1-s_{k}^{*}\right), \\
0=-\gamma \Theta_{1}^{*}+\frac{\beta_{1}}{\langle k\rangle} \sum_{k=1}^{n} k^{2} p(k) s_{k}^{*} F_{1}\left(\Theta_{1}^{*}\right), \\
0=-\gamma \Theta_{2}^{*}+\frac{\beta_{2}}{\langle k\rangle} \sum_{k=1}^{n} k^{2} p(k) s_{k} F_{2}\left(\Theta_{2}^{*}\right),
\end{array}\right.
$$


Solving the first equation of Eq.(8) yields to

$$
s_{k}^{*}=\frac{\gamma}{\gamma+k\left(\beta_{1} F_{1}\left(\Theta_{1}^{*}\right)+\beta_{2} F_{2}\left(\Theta_{2}^{*}\right)\right.} .
$$

Substituting Eq.(9) into the second and third equations of Eq.(8), one arrives at

$$
\begin{aligned}
& \Theta_{1}^{*}=\frac{\beta_{1}}{\langle k\rangle} \sum_{k=1}^{n} \frac{k^{2} P(k) F_{1}\left(\Theta_{1}^{*}\right)}{\gamma+k\left(\beta_{1} F_{1}\left(\Theta_{1}^{*}\right)+\beta_{2} F_{2}\left(\Theta_{2}^{*}\right)\right)}, \\
& \Theta_{2}^{*}=\frac{\beta_{2}}{\langle k\rangle} \sum_{k=1}^{n} \frac{k^{2} P(k) F_{2}\left(\Theta_{2}^{*}\right)}{\gamma+k\left(\beta_{1} F_{1}\left(\Theta_{1}^{*}\right)+\beta_{2} F_{2}\left(\Theta_{2}^{*}\right)\right)} .
\end{aligned}
$$

Apparently, $E_{0}=\left(s_{k}^{0}, 0,0\right)=(1,0,0)$ is always a solution of Eq.(8). Now, we consider the following two cases, namely, $\Theta_{1}^{*}=0$ or $\Theta_{2}^{*}=0$. To investigate the existence of such boundary equilibria, we define two basic reproduction number associated with each strain by

$$
\begin{aligned}
& \mathcal{R}_{01}=\frac{\beta_{1}\left\langle k^{2}\right\rangle F_{1}^{\prime}(0)}{\gamma\langle k\rangle}, \\
& \mathcal{R}_{02}=\frac{\beta_{2}\left\langle k^{2}\right\rangle F_{2}^{\prime}(0)}{\gamma\langle k\rangle} .
\end{aligned}
$$

The basic reproduction numbers of $\mathcal{R}_{0 j}(j=1,2)$ mean that the average number $\beta\left\langle k^{2}\right\rangle F_{1}^{\prime}(0) /\langle k\rangle$ of secondary infected edges associated with strain $j$ produced by an infected edge with respect to strain $j$ during its infected infectious period $1 / \gamma$ at a completely susceptible environment. Letting $\Theta_{2}^{*}=0$, then Eq.(10) can be reduced to

$$
\Theta_{1}^{*}=\frac{\beta_{1}}{\langle k\rangle} \sum_{k=1}^{n} \frac{k^{2} P(k) F_{1}\left(\Theta_{1}\right)}{\gamma+k \beta_{1} F_{1}\left(\Theta_{1}\right)}=g_{1}\left(\Theta_{1}\right)
$$

It is easy to verify that

$$
g_{1}(0)=0, g_{1}^{\prime}(0)=\frac{\beta_{1}\left\langle k^{2}\right\rangle F_{1}^{\prime}(0)}{\gamma\langle k\rangle}=\mathcal{R}_{10}, g_{1}(1)=\frac{\beta_{1}}{\langle k\rangle} \sum_{k=1}^{n} \frac{k^{2} P(k) F_{1}(1)}{\gamma+k \beta_{1} F_{1}(1)}<1
$$

Additionally,

$$
g_{1}^{\prime}\left(\Theta_{1}\right)=\frac{\beta_{1}}{\langle k\rangle} \sum_{k=1}^{n} \frac{k^{2} P(k) \gamma}{\left(\gamma+k \beta_{1} F_{1}\left(\Theta_{1}\right)\right)^{2}}>0 .
$$

Therefore, equation (14) has a unique positive solution $\Theta_{1}^{*} \in(0,1)$ provided $\mathcal{R}_{01}>1$. 
Analogous to the case $\Theta_{2}^{*}=0$, if we assume $\Theta_{1}^{*}=0$ and define

$$
\Theta_{2}^{*}=\frac{\beta_{2}}{\langle k\rangle} \sum_{k=1}^{n} \frac{k^{2} P(k) F_{2}\left(\Theta_{2}\right)}{\gamma+k \beta_{2} F_{2}\left(\Theta_{2}\right)}:=g_{2}\left(\Theta_{2}^{*}\right),
$$

then $g_{2}\left(\Theta_{2}^{*}\right)=\Theta_{2}^{*}$ has a unique positive solution $\Theta_{2}^{*} \in(0,1)$ as long as $\mathcal{R}_{02}>1$.

From what we have been discussed, we have the following theorem on the existence of equilibria of Sys.(2).

Theorem 2 Let $\mathcal{R}_{0 j}(j=1,2)$ be defined in (12) and (13), separately. Then the following claims hold.

(1) There exists a disease-free equilibrium $E_{0}$, when $\mathcal{R}_{0}=\max \left\{\mathcal{R}_{01}, \mathcal{R}_{02}\right\}<1$.

(2) There exists two equilibria, one is disease-free equilibrium $E_{0}$, the other is strain 1 dominated equilibrium $E_{1}=\left(s_{1 k}^{*}, \Theta_{1}^{*}, 0\right)$ when $\mathcal{R}_{01}>1$.

(3) There exists two equilibria, one is disease-free equilibrium $E_{0}$, the other is strain 2 dominated equilibrium $E_{2}=\left(s_{2 k}^{*}, 0, \Theta_{2}^{*}\right)$ when $\mathcal{R}_{02}>1$.

Next, we are in a position to show the stability of each feasible strain dominated equilibrium. To achieve this goal, define two invasion reproduction numbers by

$$
\begin{aligned}
& \mathcal{R}_{1}^{2}=\frac{\beta_{2}}{\gamma\langle k\rangle} \sum_{k=1}^{n} k^{2} p(k) s_{1 k}^{*} F_{2}^{\prime}(0), \\
& \mathcal{R}_{2}^{1}=\frac{\beta_{1}}{\gamma\langle k\rangle} \sum_{k=1}^{n} k^{2} p(k) s_{2 k}^{*} F_{1}^{\prime}(0) .
\end{aligned}
$$

$\mathcal{R}_{j}^{l}(j=1,2, l=1,2, j \neq l)$ indicate the ability of strain $l$ invading strain $j$ or they mean that the average number of secondary infected edges associated with strain $j$ produced by an infected edge with respect to strain $j$ during its infected infectious period at the boundary equilibrium $E_{l}$.

Lemma 1 Let $\mathcal{R}_{j}^{l}(j, l=1,2)$ be defined in (15) and (16).

(1) If $\mathcal{R}_{0} \leq 1$, then the disease-free equilibrium $E_{0}$ is globally asymptotically stable for any $\phi \in \Omega$;

(2) If $\mathcal{R}_{1}^{2}<1$, then the strain one dominated equilibrium $E_{1}$ is globally asymptotically stable for any $\phi \in \Omega \backslash \Omega_{1}$;

(3) If $\mathcal{R}_{2}^{1}<1$, then the strain one dominated equilibrium $E_{2}$ is globally asymptotically stable for any $\phi \in \Omega \backslash \Omega_{2}$.

Proof The global stability of the disease-free equilibrium comes from constructing some smart Lyapunov function, which is taken in form of

$$
V\left[\Theta_{1}, \Theta_{2}\right](t)=\Theta_{1}(t)+\Theta_{2}(t) .
$$


Calculating the derivative of $V$ along the trajectory of system (2), we obtain

$$
\begin{aligned}
\left.\frac{d V(t)}{d t}\right|_{(2)}= & \frac{d \Theta_{1}(t)}{d t}+\frac{d \Theta_{2}(t)}{d t} \\
\leq & \frac{\beta_{1}}{\langle k\rangle} \sum_{k=1}^{n} k^{2} p(k)\left[s_{k}(t)-1\right] F_{1}^{\prime}(0) \Theta_{1}(t) \\
& +\frac{\beta_{2}}{\langle k\rangle} \sum_{k=1}^{n} k^{2} p(k)\left[s_{k}(t)-1\right] F_{2}^{\prime}(0) \Theta_{2}(t) \\
& +\gamma\left(\mathcal{R}_{01}-1\right) \Theta_{1}(t)+\gamma\left(\mathcal{R}_{02}-1\right) \Theta_{2}(t) \\
\leq & \gamma\left(\mathcal{R}_{01}-1\right) \Theta_{1}(t)+\gamma\left(\mathcal{R}_{02}-1\right) \Theta_{2}(t) .
\end{aligned}
$$

Hence, $\dot{V}(t) \leq 0$ once $\mathcal{R}_{0}<1$ and the equality holds if and only if $\Theta_{1}(t)=\Theta_{2}(t)=0$ for any $t \in \mathbb{R}_{+}$. Returning to the first equation, we easily see the largest invariant set $\mathcal{M}=\{\phi \in \Omega \mid \dot{V}(t)=0\}=\left\{\phi \in \Omega \mid s_{k}(t)=1, \Theta(t)=\Theta_{2}(t)=0\right\}$. From LaSalle invariant principle, it follows that the disease-free equilibrium $E_{0}$ is globally asymptotically stable.

Alternatively, $\mathcal{R}_{0}=1$ implies that one of the following cases holds

(i) $\mathcal{R}_{01}<1$, and $\mathcal{R}_{02}=1$,

(ii) $\mathcal{R}_{01}=1$, and $\mathcal{R}_{02}<1$,

(iii) $\mathcal{R}_{01}=\mathcal{R}_{02}=1$.

The first two cases have similar features and hence we will fix one of them. If condition (i) holds, then

$$
\begin{aligned}
\left.\frac{d V(t)}{d t}\right|_{(2)} \leq & \frac{\beta_{1}}{\langle k\rangle} \sum_{k=1}^{n} k^{2} p(k)\left[s_{k}(t)-1\right] F_{1}^{\prime}(0) \Theta_{1} \\
& +\frac{\beta_{2}}{\langle k\rangle} \sum_{k=1}^{n} k^{2} p(k)\left[s_{k}(t)-1\right] F_{2}^{\prime}(0) \Theta_{2} \\
& +\gamma\left(\mathcal{R}_{01}-1\right) \Theta_{1} .
\end{aligned}
$$

Apparently, inequality (18) is semi-negative definite and the equality holds if and only if

$$
\Theta_{1}(t)=\Theta_{2}(t)=0, \text { or } \Theta_{1}(t)=0, s_{k}(t)=1 .
$$

Each above case implies that the largest invariant set $\mathcal{M}$ contains a singleton point $E_{0}$ and it is globally asymptotically stable. A similar result directly follows from the symmetric of system (2) if condition (ii) holds.

Finally, let condition (iii) hold, then

$$
\begin{aligned}
\left.\frac{d V(t)}{d t}\right|_{(2)} \leq & \frac{\beta_{1}}{\langle k\rangle} \sum_{k=1}^{n} k^{2} p(k)\left[s_{k}(t)-1\right] F_{1}^{\prime}(0) \Theta_{1} \\
& +\frac{\beta_{2}}{\langle k\rangle} \sum_{k=1}^{n} k^{2} p(k)\left[s_{k}(t)-1\right] F_{2}^{\prime}(0) \Theta_{2} .
\end{aligned}
$$

Inequality (19) preserves $\dot{V}(t) \leq 0$ and the equality holds if and only if $s_{k}(t)=1$ or $\Theta_{1}(t)=\Theta_{2}(t)=0$ for any $\bar{k}=1,2, \cdots, n$ and $t \in \mathbb{R}_{+}$. The last case has been 
discussed above. Provided $s_{k}(t)=1$ for any $t \in \mathbb{R}_{+}$, we have from the first equation of (2) that

$$
0=-\beta_{1} k F_{1}\left(\Theta_{1}\right)-\beta_{2} k F_{2}\left(\Theta_{2}\right)
$$

The properties of $F_{j}(j=1,2)$, together with the positivity of $\beta_{j}(j=1,2)$ suggest that $\Theta_{1}(t)=\Theta_{2}(t)=0$ for each $t \in \mathbb{R}_{+}$. The global result follows immediately.

In what follows, we are concerned with the global stability of each strain dominated equilibrium. To achieve this goal, let us define a Lyapunov function as follows:

$$
V_{1}\left[S, \Theta_{1}, \Theta_{2}\right](t)=\sum_{k=1}^{n} k p(k) V_{s_{k}}(t)+\langle k\rangle\left[V_{\Theta_{1}}(t)+V_{\Theta_{2}}(t)\right],
$$

where

$$
V_{s_{k}}=s_{k}-s_{k}^{*}-\ln \left(\frac{s_{k}}{s_{1 k}^{*}}\right), V_{\Theta_{1}}=\Theta_{1}-1-\ln \frac{\Theta_{1}}{\Theta_{1}^{*}}, V_{\Theta_{2}}=\Theta_{2} .
$$

Since strain one dominated equilibrium $E_{1}$ satisfies the following equations

$$
\begin{aligned}
\gamma & =\gamma s_{1 k}^{*}+\beta_{1} k s_{1 k}^{*} F_{1}\left(\Theta_{1}^{*}\right), \\
\gamma \Theta_{1}^{*} & =\frac{\beta_{1}}{\langle k\rangle} \sum_{k=1}^{n} k^{2} p(k) s_{1 k}^{*} F_{1}\left(\Theta_{1}^{*}\right) .
\end{aligned}
$$

Differentiating $V_{s_{k}}$ and $V_{\Theta_{1}}$ along trajectory of system (2) leads to

$$
\begin{aligned}
\left.\frac{d V_{s_{k}}(t)}{d t}\right|_{(2)}= & \sum_{k=1}^{n} k p(k)\left(1-\frac{s_{k}(t)}{s_{1 k}^{*}}\right) \frac{d s_{k}(t)}{d t} \\
= & -\gamma \sum_{k=1}^{n} k p(k) s_{k}\left(1-\frac{s_{k}(t)}{s_{1 k}^{*}}\right)\left(1-\frac{s_{1 k}^{*}}{s_{k}(t)}\right) \\
& -\sum_{k=1}^{n} k^{2} p(k) \beta_{2} F_{2}\left(\Theta_{2}(t)\right)\left(s_{k}(t)-s_{1 k}^{*}\right) \\
& +\sum_{k=1}^{n} k^{2} p(k) \beta_{1} s_{k}^{1 *} F_{1}\left(\Theta_{1}^{*}\right)\left[-g\left(\frac{s_{k}(t) F_{1}\left(\Theta_{1}(t)\right)}{s_{1 k}^{*} F_{1}\left(\Theta_{1}^{*}\right)}\right)\right. \\
& \left.-g\left(\frac{s_{1 k}^{*}}{s_{k}(t)}\right)+g\left(\frac{F_{1}\left(\Theta_{1}(t)\right)}{F_{1}\left(\Theta_{1}^{*}\right)}\right)\right]
\end{aligned}
$$

where

$$
g(x)=x-1-\ln x
$$


which has the properties: $g(x) \geq 0$ for all $x \geq 0$ and the equality holds if and only if $x=1$. Recalling (22), we derive

$$
\begin{aligned}
\left.\frac{d V_{\Theta_{1}}(t)}{d t}\right|_{(2)}= & \left(1-\frac{\Theta_{1}(t)}{\Theta_{1}^{*}}\right) \frac{d \Theta_{1}(t)}{d t} \\
= & \sum_{k=1}^{n} k^{2} p(k) \beta_{1} s_{1 k}^{*} F_{1}\left(\Theta_{1}^{*}\right)\left[g\left(\frac{s_{k}(t) F_{1}\left(\Theta_{1}(t)\right)}{s_{1 k}^{*} F_{1}\left(\Theta_{1}^{*}\right)}\right)-g\left(\frac{\Theta_{1}(t)}{\Theta_{1}^{*}}\right)\right. \\
& \left.-g\left(\frac{\Theta_{1}^{*} s_{k}(t) F_{1}\left(\Theta_{1}(t)\right)}{\Theta_{1}(t) s_{1 k}^{*} F_{1}\left(\Theta_{1}^{*}\right)}\right)\right] .
\end{aligned}
$$

Hence, the derivative of $V_{1}$ along the solution of system (2) takes in form of

$$
\begin{aligned}
\left.\frac{d V_{1}(t)}{d t}\right|_{(2)}= & -\gamma \sum_{k=1}^{n} k p(k) s_{k}(t)\left[g\left(\frac{s_{k}(t)}{s_{1 k}^{*}}\right)+g\left(\frac{s_{1 k}^{*}}{s_{k}(t)}\right)\right] \\
& -\sum_{k=1}^{n} k^{2} p(k) \beta_{1} s_{1 k}^{*} F_{1}\left(\Theta_{1}^{*}\right)\left[g\left(\frac{s_{k}^{1 *}}{s_{k}(t)}\right)+g\left[\frac{\Theta_{1}^{*} s_{k}(t) F_{1}\left(\Theta_{1}(t)\right)}{\Theta_{1}(t) s_{1 k}^{*} F_{1}\left(\Theta_{1}^{*}\right)}\right]\right] \\
& +\sum_{k=1}^{n} k^{2} p(k) \beta_{1} s_{1 k}^{*} F_{1}\left(\Theta_{1}^{*}\right)\left[g\left(\frac{F_{1}\left(\Theta_{1}(t)\right)}{F_{1}\left(\Theta_{1}^{*}\right)}\right)-g\left(\frac{\Theta_{1}(t)}{\Theta_{1}^{*}}\right)\right] \\
& +\sum_{k=1}^{n} k^{2} p(k) \beta_{2} s_{1 k}^{*} F_{2}^{\prime}(0) \Theta_{2}(t)\left[\frac{F_{2}\left(\Theta_{2}(t)\right)}{F_{2}^{\prime}(0) \Theta_{2}(t)}-\frac{1}{\mathcal{R}_{1}^{2}}\right]
\end{aligned}
$$

By assumption for $F_{2}$ and Proposition A.1 [27], we have that

$$
g\left(\frac{F_{1}\left(\Theta_{1}\right)}{F_{1}\left(\Theta_{1}^{*}\right)}\right) \leq g\left(\frac{\Theta_{1}}{\Theta_{1}^{*}}\right)
$$

and

$$
\frac{F_{2}\left(\Theta_{2}\right)}{\Theta_{2}} \leq F_{2}^{\prime}(0)
$$

Therefore, if $\mathcal{R}_{1}^{2}<1$, the derivative of $V$ is semi-negative definite and the equality holds if and only if

$$
s_{k}(t)=s_{1 k}^{*}, \Theta_{1}=\Theta_{1}^{*}, \Theta_{2}(t)=0 .
$$

Employing LaSalle invariant principle, we infer that the strain one dominated equilibrium $E_{1}^{*}$ is globally asymptotically stable.

Similarly, it follows from the symmetric of system (2) that the strain two dominated equilibrium $E_{2}^{*}$ is also globally asymptotically stable when $\mathcal{R}_{2}^{1}<1$. This completes the proof.

Next, we will be conducted to the existence of coexistence of equilibrium. Dividing $\Theta_{j}$ on both sides of the second and the third equation of (10), we get

$$
1=\frac{\beta_{1}}{\langle k\rangle} \sum_{k=1}^{n} \frac{k^{2} P(k) F_{1}\left(\Theta_{1}\right)}{\left(\gamma+k\left(\beta_{1} F_{1}\left(\Theta_{1}\right)+\beta_{2} F_{2}\left(\Theta_{2}\right)\right)\right) \Theta_{1}}:=f_{1}\left(\Theta_{1}, \Theta_{2}\right),
$$




$$
1=\frac{\beta_{2}}{\langle k\rangle} \sum_{k=1}^{n} \frac{k^{2} P(k) F_{2}\left(\Theta_{2}\right)}{\left(\gamma+k\left(\beta_{1} F_{1}\left(\Theta_{1}\right)+\beta_{2} F_{2}\left(\Theta_{2}\right)\right)\right) \Theta_{2}}:=f_{2}\left(\Theta_{1}, \Theta_{2}\right) .
$$

Noting that $f_{2}$ is a decreasing function of $\Theta_{1}$, Implicit Function Theorem suggests that there exists a function $h$ such that $\Theta_{1}=h\left(\Theta_{2}\right)$.

Define

$$
f_{2}\left(0, \Theta_{2}\right)=\frac{\beta_{2}}{\langle k\rangle} \sum_{k=1}^{n} \frac{k^{2} P(k) F_{2}\left(\Theta_{2}\right)}{\left(\gamma+k \beta_{2} F_{2}\left(\Theta_{2}\right)\right) \Theta_{2}} .
$$

By Theorem 2, we have known that $f_{2}\left(0, \Theta_{2}\right)=1$ has a unique solution $\Theta_{2}^{*}$. Provided $G\left(\Theta_{2}\right)=f_{1}\left(h\left(\Theta_{2}\right), \Theta_{2}\right)$, then

$$
G(0)=f_{1}\left(0, \Theta_{2}^{*}\right)=\lim _{\Theta_{1} \rightarrow 0} f_{1}\left(\Theta_{1}, \Theta_{2}^{*}\right)=\mathcal{R}_{2}^{1}>1 .
$$

On the contrary, let $H\left(\Theta_{1}\right)=f_{2}\left(h\left(\Theta_{1}\right), \Theta_{1}\right)$. Observe that

$$
H\left(\Theta_{1}^{*}\right)=f_{2}\left(\Theta_{1}^{*}, 0\right)=\lim _{\Theta_{2} \rightarrow 0} f_{2}\left(\Theta_{1}^{*}, \Theta_{2}\right)=\mathcal{R}_{1}^{2}>1 .
$$

and

$$
H(1)=\frac{\beta_{2}}{\langle k\rangle} \sum_{k=1}^{n} \frac{k^{2} P(k) F_{2}(1)}{\gamma+k \beta_{2} F_{2}(1)}<1
$$

From the monotone decreasing property of $H$, it follows that there exists a $\bar{\Theta}_{1}^{*} \in$ $\left(\Theta_{1}^{*}, 1\right)$ such that $H\left(\bar{\Theta}_{1}^{*}\right)=1$. Let $\Theta_{2}=0=h\left(\bar{\Theta}_{1}^{*}\right)$, then

$$
\begin{aligned}
G\left(\bar{\Theta}_{1}^{*}\right)=f_{1}\left(\bar{\Theta}_{1}^{*}, 0\right) & =\frac{\beta_{1}}{\langle k\rangle} \sum_{k=1}^{n} \frac{k^{2} P(k) F_{1}\left(\bar{\Theta}_{1}^{*}\right)}{\left(\gamma+k \beta_{1} F_{1}\left(\bar{\Theta}_{1}^{*}\right)\right) \bar{\Theta}_{1}^{*}} \\
& <\frac{\beta_{1}}{\langle k\rangle} \sum_{k=1}^{n} \frac{k^{2} P(k) F_{1}\left(\Theta_{1}^{*}\right)}{\left(\gamma+k \beta_{1} F_{1}\left(\Theta_{1}^{*}\right)\right) \Theta_{1}^{*}}=1 .
\end{aligned}
$$

Immediate Value Theorem, together with (27) and (28), indicates that $G$ has a unique $\tilde{\Theta}_{1}^{*} \in\left(0, \bar{\Theta}_{1}^{*}\right)$ such that $f_{1}\left(h\left(\tilde{\Theta}_{1}^{*}\right), \tilde{\Theta}_{1}^{*}\right)=1$. From what we has been discussed, we have established the coeixstence of the endemic equilibrium.

Theorem 3 If $\mathcal{R}_{1}^{2}>1$ and $\mathcal{R}_{2}^{1}>1$, then system (2) has at least one endemic equilibrium $E^{*}=\left(s_{k}^{*}, \Theta_{1}^{*}, \Theta_{2}^{*}\right)$.

Theorem 4 If $\mathcal{R}_{1}^{2}>1$ and $\mathcal{R}_{2}^{1}>1$, then the coexistence equilibrium $E^{*}$ is globally asymptotically stable for any $\phi \in \Omega \backslash\left\{\Omega_{1} \cup \Omega_{2}\right\}$.

Proof Analogous to Lemma 1, we can employ a Lyapunov function as follows

$$
V\left[s, \Theta_{1}, \Theta_{2}\right](t)=\sum_{k=1}^{n} k p(k) V_{s_{k}}(t)+\langle k\rangle\left[V_{\Theta_{1}}(t)+V_{\Theta_{2}}(t)\right],
$$


where

$$
V_{f}=f-f^{*}-f^{*} \ln \frac{f}{f^{*}}, f=s_{k}, \Theta_{1}, \Theta_{2} .
$$

Calculating the derivative of $V$ along the total trajectory of Eq.(2) takes in form of

$$
\begin{aligned}
\left.\frac{d V(t)}{d t}\right|_{(2)}= & -\gamma \sum_{k=1}^{n} k p(k) s_{k}\left[g\left(\frac{s_{k}(t)}{s_{k}^{*}}\right)+g\left(\frac{s_{k}^{*}}{s_{k}(t)}\right)\right] \\
& -\sum_{k=1}^{n} k^{2} p(k) \beta_{1} s_{k}^{*} F_{1}\left(\Theta_{1}^{*}\right)\left[g\left(\frac{s_{k}^{*}}{s_{k}(t)}\right)+g\left(\frac{\Theta_{1}^{*} s_{k}(t) F_{1}\left(\Theta_{1}(t)\right)}{\Theta_{1}(t) s_{k}^{1 *} F_{1}\left(\Theta_{1}^{*}\right)}\right)\right] \\
& +\sum_{k=1}^{n} k^{2} p(k) \beta_{1} s_{k}^{*} F_{1}\left(\Theta_{1}^{*}\right)\left[g\left(\frac{F_{1}\left(\Theta_{1}(t)\right)}{F_{1}\left(\Theta_{1}^{*}\right)}\right)-g\left(\frac{\Theta_{1}(t)}{\Theta_{1}^{*}}\right)\right] \\
& -\sum_{k=1}^{n} k^{2} p(k) \beta_{2} s_{k}^{*} F_{2}\left(\Theta_{1}^{*}\right)\left[g\left(\frac{s_{k}^{*}}{s_{k}(t)}\right)+g\left(\frac{\Theta_{1}^{*} s_{k} F_{2}\left(\Theta_{2}(t)\right)}{\Theta_{2} s_{k}^{*} F_{1}\left(\Theta_{2}^{*}\right)}\right)\right] \\
& +\sum_{k=1}^{n} k^{2} p(k) \beta_{1} s_{k}^{*} F_{2}\left(\Theta_{1}^{*}\right)\left[g\left(\frac{F_{2}\left(\Theta_{2}(t)\right)}{F_{2}\left(\Theta_{2}^{*}\right)}\right)-g\left(\frac{\Theta_{2}(t)}{\Theta_{2}^{*}}\right)\right] \\
\leq & 0,
\end{aligned}
$$

here we have used the fact that for every $t \in \mathbb{R}_{+}$,

$$
g\left(\frac{F_{j}\left(\Theta_{j}(t)\right)}{F_{j}\left(\Theta_{j}^{*}\right)}\right) \leq g\left(\frac{\Theta_{j}(t)}{\Theta_{j}^{*}}\right), j=1,2 .
$$

The equality holds if and only if $s_{k}(t)=s_{k}^{*}, \Theta_{1}(t)=\Theta_{1}^{*}, \Theta_{2}(t)=\Theta_{2}^{*}$ for all $t \in \mathbb{R}_{+}$ and $k=1,2, \cdots, n$. Hence, the largest invariant set has a unique element $E^{*}$ and so it is globally asymptotically stable by LaSalle invariant principle. This finishes the proof.

Remark 1 Theorems 3 and 4 show that the coexistence equilibrium uniquely exists. From epidemiological view of points, if the mutually invasive abilities of two strains are strong enough, the two strain may attain certain equivalence and thus the coexistence phenomenon happens.

\section{Applications}

In this section, we will give some applications for clarifying the nonlinear incidence rate. Let us define the basic reproduction numbers associated with each strain by

$$
\mathcal{R}_{0 j}=\frac{\beta_{j}\left\langle k^{2}\right\rangle}{\gamma\langle k\rangle}, j=1,2, j .
$$

In the section, we assume that system (2) has two boundary equilibria $E_{1}=$ $\left(s_{1 k}^{*}, \Theta_{1}^{*}, 0\right)$ and $E_{2}=\left(s_{2 k}^{*}, 0, \Theta_{2}^{*}\right)$, 


\subsection{The bilinear incidence rate}

If we select

$$
F_{j}\left(\Theta_{j}\right)=\Theta_{j}
$$

We have noted that $F_{j}^{\prime}\left(\Theta_{j}\right)=1>0$ and $F_{j}^{\prime \prime}\left(\Theta_{j}\right)=0$. Hence, the first two conditions of assumption for $F_{j}$ are satisfied. However, $\frac{F_{j}\left(\Theta_{j}\right)}{\Theta_{j}}=1$ is constant and so it doesn't satisfy condition (3) for $F$. The endemic equilibrium $E^{*}=\left(s_{k}^{*}, \Theta_{1}^{*}, \Theta_{2}^{*}\right)$ exists holds if and only if $\beta_{1}=\beta_{2}$. Adversely, $s_{j k}^{*}(j=1,2)$ in the associated equilibrium $E_{j},(j=$ $1,2)$ satisfy the following equations

$$
\frac{1}{\langle k\rangle} \sum_{k=1}^{n} k^{2} p(k) s_{j k}^{*}=\frac{\gamma}{\beta_{j}}, j=1,2 .
$$

Hence, the invasion reproduction numbers associated with each strain is defined by

$$
\mathcal{R}_{j}^{l}=\frac{\beta_{l}\left\langle k^{2} s_{j k}^{*}\right\rangle}{\gamma\langle k\rangle}=\frac{\mathcal{R}_{l 0}}{\mathcal{R}_{j 0}}, l=1,2, j=1,2, j \neq l .
$$

According to Lemma 1, the competitive exclusion principle holds. From biological view of points, if the process of individual contacts is a linear process or each individual is independent, then individuals infected one strain dominate and other infectives will be eradicated in a region.

Corollary 1 Let $\mathcal{R}_{0 j}$ be defined in (29).

(1) If $\mathcal{R}_{0} \leq 1$, then $E_{0}$ is globally asymptotically stable for any $\phi \in \Omega$;

(2) If $\mathcal{R}_{01}>1$, and $\mathcal{R}_{20}<\mathcal{R}_{10}$, then $E_{1}$ is globally asymptotically stable for any $\phi \in \Omega \backslash\left\{\Omega_{1} \cap \Omega_{2}\right\}$

(3) If $\mathcal{R}_{02}>1$, and $\mathcal{R}_{10}<\mathcal{R}_{20}$, then $E_{2}$ is globally asymptotically stable for any $\phi \in \Omega \backslash\left\{\Omega_{1} \cap \Omega_{2}\right\} ;$

Corollary 1 can be considered as a generalized the results in Sect. 2.1 in [17] which suggests that the competitive exclusion principle exists locally.

\subsection{The saturation incidence rate}

If we take

$$
F_{j}\left(\Theta_{j}\right)=\frac{\Theta_{j}}{1+\alpha_{j} \Theta_{j}}, j=1,2
$$

then $F_{j}^{\prime}(0)=1$. Assume that

$$
\mathcal{R}_{j}^{l}=\frac{\beta_{l}\left\langle k^{2} s_{j k}^{*}\right\rangle}{\gamma\langle k\rangle}, l=1,2, j=1,2, j \neq l,
$$


where

$$
\left\langle k^{2} s_{j k}^{*}\right\rangle=\sum_{k=1}^{n} k^{2} p(k) s_{j k}^{*}
$$

From Lemma 1 and Theorem 4, it follows that

Corollary 2 Let $\mathcal{R}_{0 j}$ and $\mathcal{R}_{j}^{l}(j, l=1,2)$ be defined in (29).

(1) If $\mathcal{R}_{0} \leq 1$, then $E_{0}$ is globally asymptotically stable for any $\phi \in \Omega$;

(2) If $\mathcal{R}_{01}>1$, and $\mathcal{R}_{1}^{2}<1$, then $E_{1}$ is globally asymptotically stable for any $\phi \in \Omega \backslash\left\{\Omega_{1} \cap \Omega_{2}\right\} ;$

(3) If $\mathcal{R}_{02}>1$, and $\mathcal{R}_{2}^{1}<1$, then $E_{2}$ is globally asymptotically stable for any $\phi \in \Omega \backslash\left\{\Omega_{1} \cap \Omega_{2}\right\} ;$

(4) If $\mathcal{R}_{1}^{2}>1$ and $\mathcal{R}_{2}^{1}>1$, then $E^{*}$ is globally asymptotically stable for any $\phi \in$ $\Omega \backslash\left\{\Omega_{1} \cup \Omega_{2}\right\}$.

Corollary 2 can be considered as an extended version of Theorems 3.4 and 4.1 [21]. Interestingly, we clarify the condition of coexistence equilibrium and strictly prove the global stability of such equilibrium. Furthermore, we note that the last conditions of Corollary 2 are more concise than the conditions of Theorem 4.1 in [21].

\subsection{The nonlinear connectivity}

On the other hand, if we take $k=\varphi(k)$ and $k F_{j}\left(\Theta_{j}\right)=\tilde{F}_{k j}\left(\Theta_{j}\right), j=1,2 ; k=$ $1,2, \cdots, n$, then

$$
\mathcal{R}_{0 j}=\frac{\left\langle\varphi(k) F_{k j}^{\prime}(0)\right\rangle}{\gamma\langle k\rangle}, \mathcal{R}_{j}^{l}=\frac{\left\langle\varphi(k) s_{j k}^{*} F_{k l}^{\prime}(0)\right\rangle}{\gamma\langle k\rangle}, j=1,2, l=1,2, j \neq l .
$$

Again using Lemma 1 and Theorem 4, we have concluded the competitive, exclusion and coexistence results as in Corollary 2, which can be considered as a further result of Theorem 3.4 and Theorem 4.1 in [22] from a global perspective. To visually insight into the dynamics of model (2), we are conducted to numerical simulation to show our theoretical results. First, we take the size of nodes $N=1000$ and the average network $\langle k\rangle=3$. Then let us generate a configure network. Now, we fix recovered rate $\gamma_{1}=\gamma_{2}=0.01$.

First, we choose $F_{j}\left(\Theta_{j}\right)=\Theta_{j}(j=1,2)$ and $\beta_{1}=0.003, \beta_{2}=0.0028$, then $\mathcal{R}_{01}=1.17>\mathcal{R}_{02}=1.0920>1$. Corollary 1 suggests that the infected edges with strain one dominates and the other infected edges will die out (see Fig. 1). Figure 1b shows the bifurcation diagram of system (2) when $\alpha_{1}=\alpha_{2}=0$. In Fig. 1b, system (2) has only disease-free equilibrium and it is globally asymptotically stable in the green region. Moreover, it has a strain one dominance equilibrium $E_{1}^{*}$ in yellow area and a strain two dominance equilibrium $E_{2}^{*}$ in the purple region. Second, provided $\alpha_{1}=$ $\alpha_{2}=0.01$ and $\beta_{1}=0.0028, \beta_{2}=0.003$, then $\mathcal{R}_{02}=1.17>\mathcal{R}_{01}=1.0920>1$ and $\mathcal{R}_{2}^{1}=0.9345<1$. Figure 2 indicates that infected edges with strain two dominates 


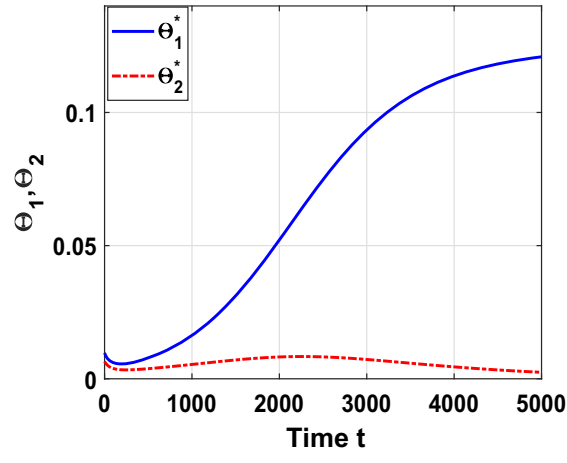

(a)

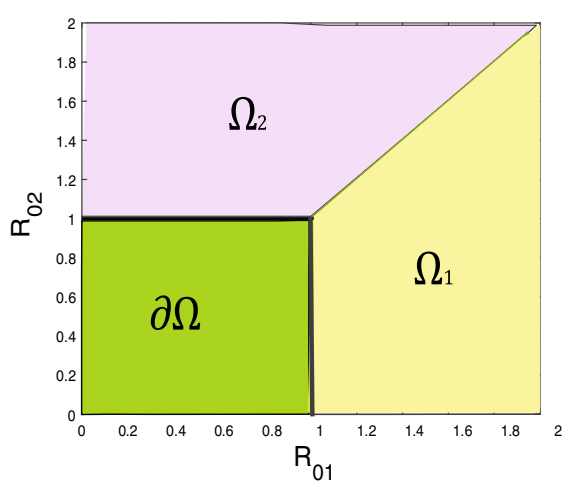

(b)

Fig. 1 a The evolution of the infected edges associated with each strain. b Plots of invasion regions in the plane of $\left(\mathcal{R}_{01}, \mathcal{R}_{02}\right)$

and infected edges die out (see Fig. 2a). Enlarging $\alpha_{2}=1.0$, then $\mathcal{R}_{1}^{2}=1.0722>1$ and $\mathcal{R}_{2}^{1}=1.005>1$. Corollary 2 infers that system (2) has a coexistence equilibrium and it is globally asymptotically stable (see Fig. 2b). Conversely, Corollary 2 infers that $\mathcal{R}_{1}^{2}, \mathcal{R}_{2}^{1}$ and $\mathcal{R}_{0}$ separate the invariant set $\Omega$ into an eradication region, two competitive regions and a coexistence area. Figure 3 displays that $\partial \Omega$ is the eradication area of the disease; $\Omega_{j}(j=1,2)$ denotes the strain $j$ dominated regions, which means that if the parameters are taken in that region, strain $j$ must compete the other strain and make it burn out; $\Omega_{0}$ represents the coexisting region of both strains, which area is gradually decreasing as values of $\alpha_{j}(j=1,2)$ increase.

Figure 4 illustrates the evolution of the two strains associated with parameters $\alpha_{j}(j=1,2)$. We have seen that the two strains undergo a switching phenomena, i.e, the competitive exclusion, coexistence and competitive exclusion. If $\beta_{j}(j=1,2)$ is larger, then the associated strain will dominate and compete the other at the initial

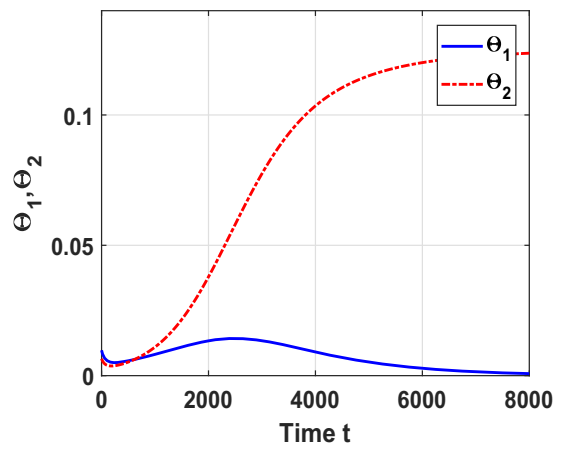

(a)

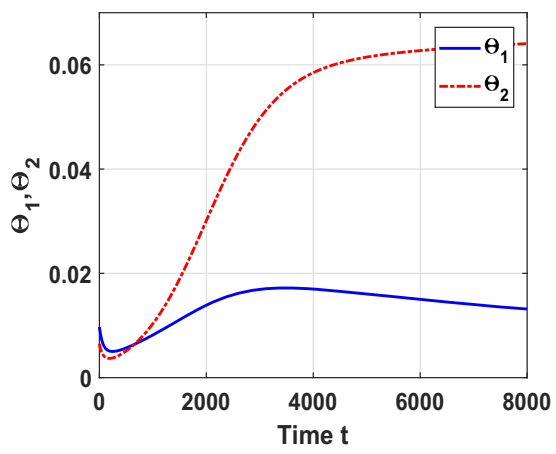

(b)

Fig. 2 The evolution of the infected edges associated with each strain a With $\alpha_{1}=\alpha_{2}=0.01$. b With $\alpha_{1}=0.01, \alpha_{2}=1.0$ 


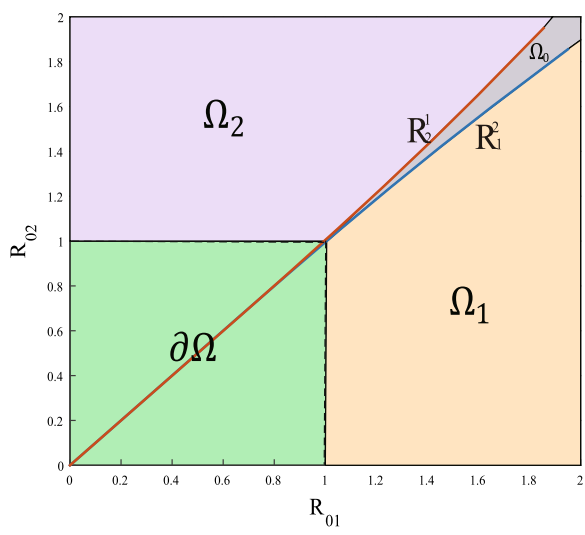

(a)

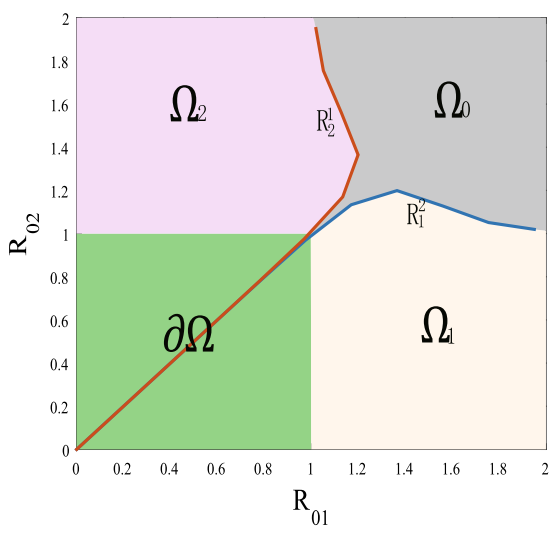

(b)

Fig. 3 Plots of invasion regions in the $\left(\mathcal{R}_{01}, \mathcal{R}_{02}\right)$. a Parameters are taken as $\alpha_{1}=\alpha_{2}=20$. b Parameters are taken as $\alpha_{1}=\alpha_{2}=0.01$

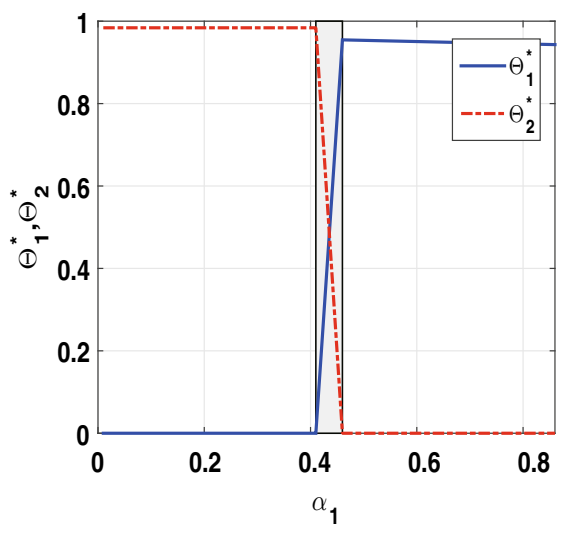

(a)

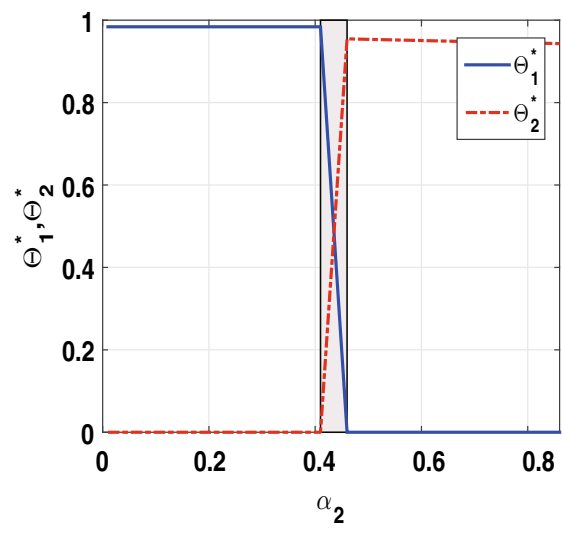

(b)

Fig. 4 Plots of endemic steady state $\Theta_{j}^{*}$ associated delay effects $\alpha_{j}(j=1,2)$. a $\beta_{1}=0.1, \beta_{2}=0.2$ and $\alpha_{2}=0.01$. b $\beta_{1}=0.2, \beta_{2}=0.1$ and $\alpha_{1}=0.01$

phase; as the values of $\alpha_{j}(j=1,2)$ increases from 0.41 to 0.46 , the two strains coexist; when $\alpha_{j}(j=1,2)$ continuously increase, the dominant relationship is reversed.

To catch the sight of the effects on different networks, we select $p(k)=m \times$ $k^{-2.4}(k=1,2, \cdots, n)$ where $m$ is a coefficient for $\langle k\rangle=3$. Obviously, such action generates a BA network [17]. Figure 5 shows the evolutions of infected edges with each strain on a stochastic network and a BA network. We have found that the densities of infected edges on a stochastic network are bigger than those on a BA network. Hence, the topology of the network plays an key role in characterizing the disease transmission. 


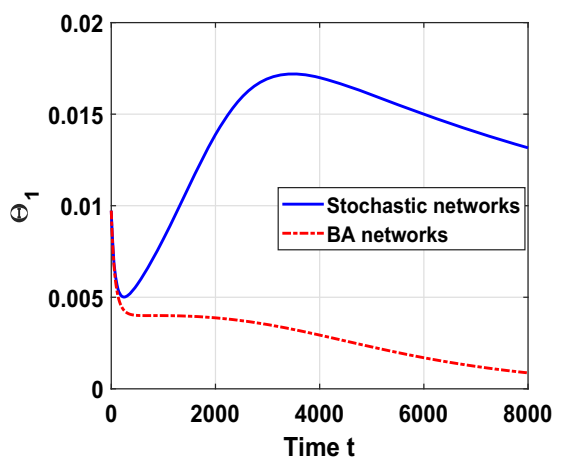

(a)

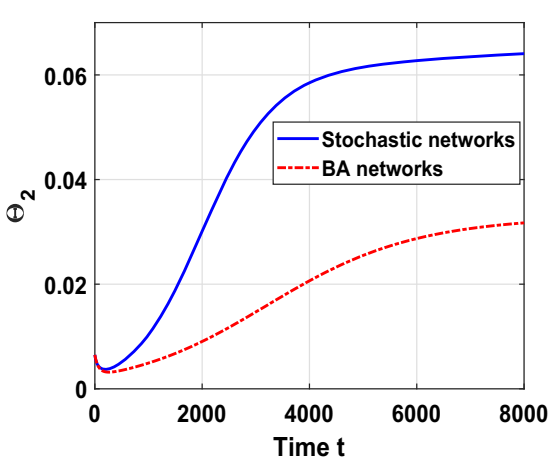

(b)

Fig. 5 The evolutions of infected edges on different networks. a The evolution of infected edges with strain one; $\mathbf{b}$ The evolution of infected edges with strain two

\section{Discussion}

Competitive exclusion and coexistence phenomena have always been the focuses of studying multi-strain models. Once the competitive exclusion principle holds, the strain with the largest reproduction number will compete the other strains. Such principle in most of existing results [21] and [22] was established from local perspectives. Mathematical speaking, such results hold only and if only one needs to pick up initial values of model (2) closely to the dominance equilibria nor a global space. In fact, the competitive exclusion principle should be established in a global sense as seen in Sect. 8.1.3 [25] and Theorem 6.10 in [18]. On the contrary, many mechanisms, such as mutation, coinfection, superinfection and nonlinear incidence etc., have been illustrated to lead to the coexistence phenomena for multi-strain models on homogeneous environments [11-15] or on complex networks [21, 22, 28]. Generally, the conditions of coexistence are tedious [21] or the proof process is vague [22]. In this paper, we have adopted the Implicit Function Theorem to definitely clarify those conditions and pointed out the uniqueness of that equilibrium as seen Theorems 3 and 4 .

Nonlinear incidence rate plays a significant role in resulting in the coexistence of multi-strain. In our model, parameters $\alpha_{j}(j=1,2)$ approximately characterize the nonlinear properties. If the values of $\alpha_{j}(j=1,2)$ are small enough, the incidence rates closely approximate to be bilinear ones. On the other hand, we have noticed that $0 \leq \Theta_{j}(t) \leq 1$ for all $t \in \mathbb{R}_{+}$. Hence, if $\alpha_{j}(j=1,2)$ are large enough, $\alpha_{j} \Theta_{j}(t)$ dominate by the values of $\alpha_{j}(j=1,2)$ and those incidence rates go back to bilinear ones. In both cases, competitive and exclusion phenomenon occurs. The coexistence phenomenon takes place when $\alpha_{j}(j=1,2)$ are taken as appropriated values as seen the coexistence zone in Fig. 4.

From epidemiological view of points, the nonlinear incidence rate $F_{j}\left(\Theta_{j}\right)(j=$ $1,2)$, considered as the contacts of individuals are strongly dependent, such as familial clusters or sociality bunching behaviors, is one of important factors leading to coexistence phenomenon of multiply strains. Undoubtedly, this phenomena increases the heterogeneity of infections and enhances the difficulties of diseases control. Pub- 
lic health governments and policymakers should take more attention on dependent conductivities among individuals for curbing multi-strain epidemics.

Model (2) can be considered as extended versions of models with nonlinear incidence rates $[21,22]$. Theoretical results give a strong proof of competitive exclusion phenomena from global points of view. The coexistence equilibrium uniquely and globally exists when the invasion abilities of both strains are strong enough.

Acknowledgements This work is partially supported by the National Natural Science Foundation of China (No.12001339, No.61573016), the Shanxi Province Science foundation (20210302123454), and the Shanxi Province Science Foundation for Youths (No. 201901D211413).

\section{References}

1. Butler, G.J., Hsu, S.B., Waltman, P.: Coexistence of competing predators in a chemostat. J. Math. Biol. 17, 133-151 (1983)

2. Levin, S.A.: Community equilibria and stability, and an extension of the competitive principle exclusion. Amer. Naturalist 104, 413-423 (1970)

3. Anderson, R.M., May, R.M.: Co-evolution of host and parasites. Parasitology 85, 411-426 (1982)

4. May, R.M., Anderson, R.M.: Parasite-host coevolution. Parasitology 100, S89-S101 (1990)

5. Beck, K.: Co-evolution. Mathematical aspects of host-parasite interactions. J. Math. Biol. 19, 63-77 (1984)

6. Castillo-Chavez, C., Huang, W., Li, J.: Competitive exclusion in gonorrhea models and other sexually transmitted diseases. SIAM J. Appl. Math. 56, 494-508 (1996)

7. Castillo-Chavez, C., Huang, W., Li, J.: Competitive exclusion and coexistence of multiple strains in an SIS STD model. SIAM J. Appl. Math. 59(5), 1790-1811 (1999)

8. Bremermann, H.J., Thieme, H.R.: A competitive exclusion principle for pathogen virulence. J. Math. Biol. 27, 179-190 (1989)

9. Cai, L., Li, X.Z., Martcheva, M.: Competitive exclusion in a vector-host epidemic model. J. Biol. Dynam. 7, 47-67 (2013)

10. Dang, Y.X., Li, X.Z., Martcheva, M.: Competitive exclusion in a multi-strain immuno- epidemiological flu model with environment transmission. J. Biol. Dynam. 10, 416-456 (2016)

11. Qian, D.L., Li, X.Z., Ghosh, M.: Coexistence of the strains induced by mutation. Inter. J. Biomath. 5, 1-25 (2012)

12. Ianelli, M., Martcheva, M., Li, X.Z.: Strain replacement in an epidemic model with superinfection and perfect vaccination. Math. Biosci. 195, 23-46 (2005)

13. Andreasen, V., Lin, J., Levin, S.: The dynamics of cocirculation influenza strains conferring partial cross-immunity. J. Math. Biol. 35, 825-842 (1997)

14. Meskaf, A., Khyar, O., Danane, J., Allali, K.: Global stability of a two-strain epidemic model with non-monotone incidence rate. Chaos, Solitions and Fractals 133, 109647 (2020)

15. Minayev, P., Ferguson, N.: Improving the realism of deterministic multi-strain models: implications for modelling influenza A. J. R. Soc. Interface 6, 509-518 (2009)

16. Karrer, B., Newman, M.E.J.: Competing epidemic on complex networks. Phy. Rev. E 84, 036106 (2011)

17. Wu, Q.C., Fu, X.C., Meng, Y.: Epidemic thresholds in a heterogenous population with competing strains. Chin. Phys. B 20(4), 046401 (2011)

18. Yang, J., Kuniya, T., Luo, X.: Competitive exclusion in a multi-strain SIS epidemic model on complex networks. Electron. J. Different. Equat. 6, 1-30 (2019)

19. Wu, Q., Small, M., Liu, H.: Superinfection behaviors on scales-free networks with competing strains. J. Nonlinear Sci. 23, 113-127 (2013)

20. Wu, Q.C., Fu, X.C.: Dynamics of competing strains with saturated infectivity and mutation on networks. J. Biol. Syst. 24, 1-17 (2016)

21. Yang, J., Li, C.: Dynamics of a competing two-strain SIS epidemic model on complex networks with a saturating incidence rate. J. Phys. A 49, 215601 (2016) 
22. Cheng, X., Wang, Y., Huang, G.: Dynamics of a competing two-strain SIS epidemic model with general infection force on complex networks. Nonlinear Anal.:RWA 59, 103247 (2021)

23. Chen, S., Wang, K., Sun, M., Fu, X.: Spread of competing viruses on heterogeneous networks. Phil. Trans. R. Soc. A 375, 20160284 (2017)

24. Lv, J.P., Jin, Z.: Multistrain edge-based compartmental model on networks. Math Method Appl. Sci. 42, 1529-1552 (2019)

25. Martcheva, M.: An Introduction to Mathematical Epidemiology. Springer, New York (2015)

26. Wang, X., Yang, J.: Dynamical analysis of a mean-field vector-borne diseases model on complex networks: An edge based compartmental approach. Chaos 30, 013103 (2020)

27. Sigdel, R.P., McCluskey, C.C.: Global stability for an sei model of infectious disease with immigration. Appl. Math. Comput. 243, 684-689 (2014)

28. Li, X.Z., Yang, J.Y., Martcheva, M.: Age Structured Epidemic Modelling. Springer, Switzerland (2020)

Publisher's Note Springer Nature remains neutral with regard to jurisdictional claims in published maps and institutional affiliations. 